\title{
Incentive preference as a function of mode of training, sucrose concentration, and water deprivation in the rat
}

\author{
JOHN FISK and JEROME S. COHEN \\ University of Windsor, Windsor, Ontario, Canada N9B $3 P_{4}$
}

\begin{abstract}
Highly and moderately water-deprived animals $(23.5 \mathrm{~h}$ and $18 \mathrm{~h}$, respectively) were exposed to $8 \%$ sucrose and water or $20 \%$ sucrose and water at fixed positions in a two-choice discrimination apparatus. Half the animals in each group received a series of alternating free- and forced-choice trials. The other animals received a series of forced-choice trials prior to a free-choice preference test. A retesting procedure was carried out in which deprivation levels were switched for all animals. Neither mode of training, exposure to incentive, nor concentration of sucrose reliably affected incentive preference. Deprivation level, however, was related to incentive preference. Only animals that were initially moderately deprived developed reliable and consistent preferences to sucrose. Highly deprived animals also developed reliable sucrose preferences after being switched to moderate water deprivation during retesting.
\end{abstract}

Beck and Bidwell (1974) exposed highly waterdeprived $(23.5 \mathrm{~h})$ and nondeprived rats to sucrose $(8 \%)$ and water at fixed positions for a lengthy series of alternating free- and forced-choice trials. Nondeprived animals developed high sucrose preferences, while the water-deprived animals displayed no incentive preferences. These results replicated earlier findings for animals not given such prolonged exposure to each incentive (Beck \& Nash, 1969; Beck, Nash, Viernstein, \& Gordon, 1972; Beck, Self, \& Carter, 1965). Cohen and Oöstendorp (1976) and Cohen, Oöstendorp, and Ross (in press) carried out experiments similar in design to Beck and Bidwell's (1974) but found reliable sucrose preferences in highly and moderately water-deprived animals $(22.5$ and $18 \mathrm{~h}$ ). These recent studies differed from Beck and Bidwell's work in that animals were exposed to a higher concentration of sucrose (20\%) and received a series of forced-choice trials prior to freechoice tests for preference. The present study was carried out to determine if these differences in mode of training and sucrose concentration could have caused such contradictory findings.

\section{METHOD}

\section{Subjects}

Ninety-six male albino rats (Wistar strain) from the breeding colonies of Woodlyn Farms, Guelph, Ontario, Canada, approximately 80 days of age at the time of pretraining, were used. During the course of the study, two animals failed to run in the

The present study is based on a master's thesis submitted to the Department of Psychology, University of Windsor by the first author. The authors wish to thank Dr. M. Adelman, Dr. T. Hirota, and Dr. B. Rourke for their valuable criticisms of the present research. Requests for printouts should be sent to J.S. Cohen, Department of Psychology, University of Windsor, Windsor, Ontario, Canada N9B 3P4. apparatus and had to be replaced by animals from the same breeding stock.

\section{Apparatus and Materials}

A simultaneous two-choice discrimination apparatus consisting of a start chamber, $\mathrm{V}$-shaped decision chamber, and two goal chambers was used. It was identical to that employed by Cohen and Oöstendorp (1976). Entrance to each goal chamber was through a $10 \times 10 \mathrm{~cm}$ doorway which could be blocked by a door. Sucrose solutions ( $8 \%$ and $20 \%$ ) were made with tap water by weight/volume. Tap water also served as the nonsweetened liquid. All liquids were maintained at room temperature during an experimental session. Sucrose solutions were refrigerated when not used.

\section{Procedure}

The procedure consisted of four phases: pretraining, training, testing, and deprivation shift-retesting. Animals were randomly divided into two deprivation groups: high and moderate. Highdeprivation animals received $.5 \mathrm{~h}$ water in their home cages following each experimental session (22.5 h deprivation). Moderate-deprivation animals received $6 \mathrm{~h}$ water following each experimental session (18 $\mathrm{h}$ deprivation). All animals received food ad lib when not in the discrimination box. Each deprivation group was further divided into two concentration groups: $8 \%$ and $20 \%$ sucrose concentration. Animals were allowed to explore the apparatus and drink their appropriate sucrose concentration in the goal chamber for $5 \mathrm{~min} /$ day for 3 days. For the next 4 days each animal was given four forced-choice trials in a single alternation sequence. A door blocked one of the goal chamber entrances. In this and all succeeding trials, each animal was allowed a 10 -sec drink of the reinforcement on each trial. A spaced trial procedure was also introduced in this phase in that each animal had to wait in its home cage until all other animals had completed the trial before receiving the next trial. Squads of 16 animals were run in this manner, creating ITIs of 10-12 min.

In the training phase all animals were exposed to sucrose and water at fixed positions in the apparatus. Animals within each deprivation-concentration group were divided into two training groups. One group received a series of alternating free- and forced-choice trials. Trials 1,3 , and 5 were free choice in that both doorways were open. Trials 2,4 , and 6 were forced choice. in that one of the doorways was blocked. On a forced-choice trial an animal was forced to go to the opposite side from that 
chosen on the preceding free-choice trial. This procedure insured that each animal experienced both incentives for an equal number of trials (three) on each day. Animals trained under these conditions were labeled alternation groups. All animals received 10 days of this type of training. The second group of animals received only six forced-choice trials per session for 10 days. These animals were labeled as forced-choice groups. The pattern of forced choices for each animal was determined by a counterbalanced yoked control procedure. Half the forcedchoice animals were yoked to animals within the same deprivation level in the alternation group, while the remaining forcedchoice animals were yoked to alternation animals on the opposite deprivation level. Therefore, any pattern of responding to incentives due to deprivation and alternation training was also experienced by forced-choice animals. Thus, eight independent deprivation/training/concentration groups, each containing 12 animals, were formed: high/alternation $/ 8 \%$, high/forced-choice/ $8 \%$, moderate/alternation $/ 8 \%$, moderate/forced-choice $/ 8 \%$, high/ alternation/ $20 \%$, high/forced-choice/ $20 \%$, moderate/alternation/ $20 \%$, moderate/forced-choice $/ 20 \%$.

During the testing phase, all rats received six trials per day for 4 days under the alternation pattern used for the alternation groups.

In the last phase animals were shifted to the opposite deprivation state. Animals remained in their home cages for 3 days in order to adapt to their new deprivation schedules. Following this period, animals were retested for their incentive preference in the 4-day alternation procedure described above.

\section{RESULTS}

Percent of choices for sucrose solution for each rat over blocks of two daily sessions (six free-choice trials) were transformed into 2 arcsin $\sqrt{\mathrm{X}}$ scores, as suggested by Winer (1971). Analyses of variance were performed on the transformed data but results are described as percent scores for the sake of clarity (see Figure 1). Separate analyses of variance were carried out for the training phase [deprivation by sucrose concentration by blocks (repeated measure) for alternation animals only] and for each test phase (deprivation by sucrose concentration by training mode by blocks). Two types of individual comparisons were used where warranted. In the first comparison choice, scores were compared to a transformed .50 score in order to determine if animals were responding significantly differently from chance or no preference. Single sample t tests were employed for this comparison. The second set of comparisons determined the significance of any differences in preferences between groups and blocks. Newman-Keuls procedures were employed in both types of comparisons in order to attenuate Type I error due to multiple comparisons.

As seen in Figure 1, deprivation level primarily influenced incentive preference. Only moderately deprived animals developed reliably high preferences for sucrose (80\%-90\%) over all phases including the retest phase, where such animals were maintained on high water deprivation. Highly water-deprived animals failed to display consistent incentive preferences during training and testing. On retest, however, these animals did display some increase in preference to $8 \%$ sucrose and a large increase in preference to $20 \%$ sucrose on Block 2 . Neither sucrose concentration nor mode of training appeared to affect preferences for incentives in any consistent manner.

Results from statistical analyses confirmed the above observations. Highly significant effects for deprivation were found for test and retest preferences $[\mathrm{F}(1,88)=$ $24.07,14.72$, respectively, $\mathrm{p}<.01]$. A significant Deprivation by Blocks interaction was also found during
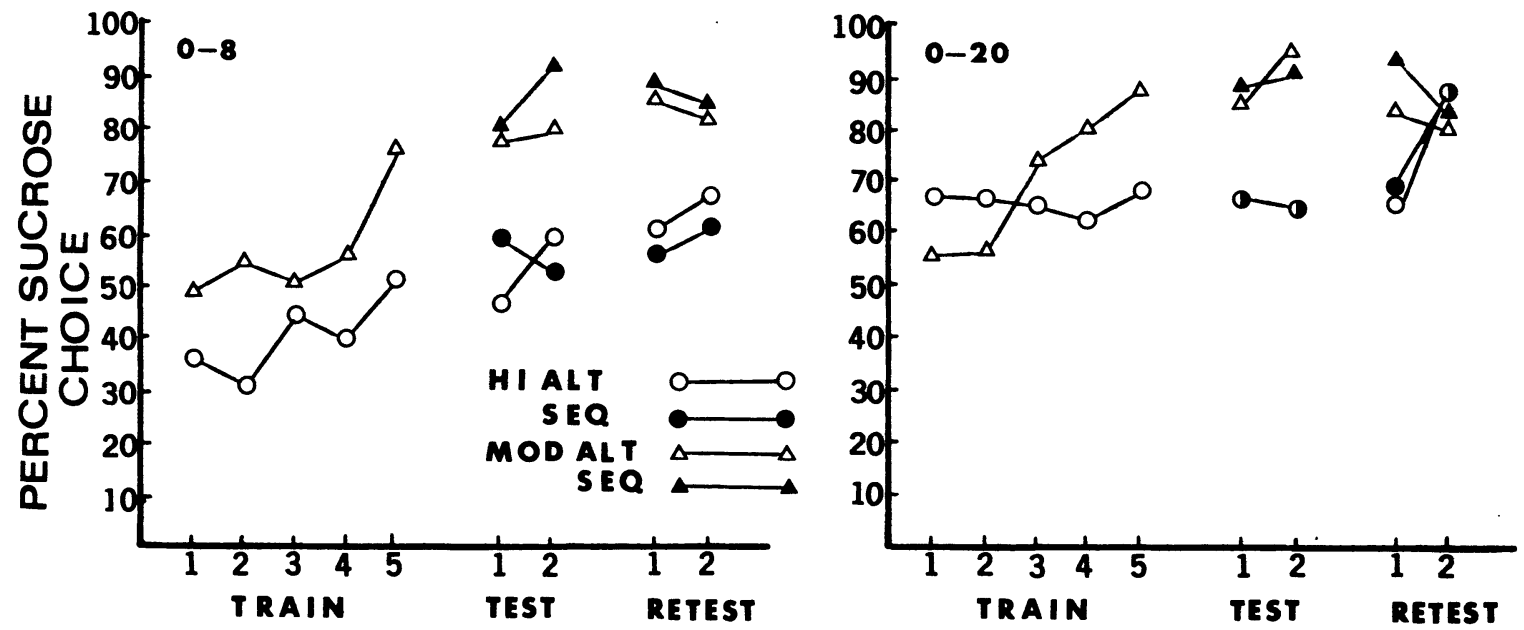

\section{TRIAL BLOCKS}

Figure 1. Percent preferences for each sucrose concentration for high-deprivation/alternation (HI-ALT), highdeprivation/forced-choice (HI-SEQ), moderate-deprivation/alternation (MOD-ALT), and moderate/forced-choice (MOD-SEQ) animals in each sucrose concentration group (water and 8\% sucrose-0-8, water and 20\% sucrose-0-20). Animals during retesting were shifted to the opposite drive although symbols for each group remain the same. 
retesting $[F(1,88)=19.67, p<.01]$. Significant blocks effects were found during training for the alternation groups: $[\mathrm{F}(4,176)=6.55, \mathrm{p}<.05]$ and during retesting for all animals $[F(1,88)=4.88, p<.05]$. A four-factor interaction occurred during testing $[\mathrm{F}(1,88)=4.36$, $\mathrm{p}<.05]$. Individual comparisons based on these last two effects did not yield information that substantially changed the basic pattern of results due to deprivation level or deprivation and blocks. Therefore, only comparisons primarily based on deprivation and deprivation by blocks effects are reported below in the interests of clarity and space.

The lack of any deprivation effects for the alternation groups during training was due to the fact that such effects developed during the final portion of that phase. Moderately deprived animals developed preferences to $20 \%$ sucrose $(\mathrm{p}<.05)$ by Block 4 . A similar increase in preference was observed for $8 \%$ sucrose on Block 5 , which, although a significant increase above earlier preferences $(p<.05)$, just barely failed to reach a critical level (.77 observed, .79 critical level above nonpreference). Highly deprived animals failed to significantly depart from nonpreference scores.

During testing and retesting, moderately deprived animals significantly preferred either concentration above nonpreference on all blocks $(\mathrm{p}<.05$ in Test Blocks 1 and 2 to $8 \%$ sucrose, $p<.01$ in all other conditions). Deprivation shift did not significantly affect these animals. Highly deprived animals continued to prefer sucrose solutions not significantly above .50 and significantly below levels displayed by moderately deprived rats $(p<.05)$ during testing. When these animals were shifted into moderate deprivation (retest), however, they rapidly developed significant preferences to each concentration ( $\mathrm{p}<.05$ to $8 \%$ on Retest Block $2, \mathrm{p}<.01$ to $20 \%$ on both retest blocks). The high-moderate animals continued to significantly prefer $8 \%$ sucrose (both blocks) and $20 \%$ sucrose (Block 1) less than did the moderate-high animals $(\mathrm{p}<.01)$. However, the highmoderate animals significantly increased their preference for $20 \%$ sucrose on Block 2 to equivalent levels of the moderate-high group.

\section{DISCUSSION}

The present results replicate earlier findings reported by Beck and Bidwell (1974). Highly water-deprived animals failed to develop reliable preferences to sucrose as had been found by Cohen and Oöstendorp (1976). When such animals were shifted to moderate water deprivation, however, they began to develop reliable preferences to sucrose. Neither mode of training nor incentive exposure (forced vs alternating free and forced choice) $(8 \%$ or $20 \%)$ affected incentive preference as expected. Only a marginal effect of sucrose concentration was found. Preferences to $20 \%$ sucrose were slightly more stable than to $8 \%$ sucrose during training for moderate-alternation animals and during retesting for high shifted to moderate deprivation animals.

Two other differences between Beck and Bidwell's (1974) and Cohen and Oüstendorp's (1976) experiments that might have affected sucrose preference in highly water-deprived rats were number of daily trials and mode of testing. Cohen and Oöstendorp presented more daily trials than Beck and Bidwell (12 vs 6). In both studies forced-choice trials occurred within the free-choice preference phases. Position of the available incentive during the forced-choice trials was independent of prior free-choice behavior in Cohen and Oöstendorp's study but was determined by such behavior in Beck and Bidwell's and the present study. These two variables may be potentially important factors for further investigation.

\section{REFERENCES}

BECK, R. C., \& Bidwell, L. D. Incentive motivational properties of sucrose and saccharin under different deprivation conditions. Learning and Motivation, 1974, 5, 328-335.

BECK, R. C., \& NASH, R. Thirsty rats do prefer sucrose. Psychonomic Science, 1969, 15, 19-20.

Beck, R. C., Nash, R., Viernstein, L., \& Gordon, L. Sucrose preferences of hungry and thirsty rats as a function of duration of stimulus presentation. Journal of Comparative and Physiological Psychology, 1972, 78, 40-50.

Beck, R. C., Self, J. S., \& Carter, D. J. Sucrose preference thresholds for satiated and water deprived rats. Psychological Reports, 1965, 16, 901-905.

Cohen, J. S., \& Oöstendorp, A. Incentive approach behavior as a function of water deprivation and presentation in the albino rat. Bulletin of the Psychonomic Society, 1976, 8, 381-384.

Cohen, J. S., Oöstendorp, A., \& Ross, W. Incentive preference as a function of water deprivation and location of discriminative stimuli. Bulletin of the Psychonomic Society, in press.

WINER, B. J. Statistical principles in experimental design. (2nd ed.) New York: McGraw-Hill, 1971.

(Received for publication February 7, 1977.) 